\title{
HYDROGEN ATOM IN 2S STATE IN A LASER FIELD ${ }^{\dagger}$ \\ UDC $546.11: 54-188+621.375 .826$
}

\author{
Svetlana Vučić* \\ Institute of Physics, University of Belgrade, PO Box 57, 1001 Belgrade, Serbia
}

\begin{abstract}
The hydrogen atom in the $2 s$ state exposed to a linearly polarized laser field is studied by using the non-perturbative non-Hermitian Floquet theory. The electronic density of the quasi-energy $H(2 s)$ state versus the electron coordinate is analyzed. We conclude that the decay of an atom in a low-intensity non-resonant laser field occurs from the asymptotically distant part of the initial state. On the other hand, the process of electron emission in a resonant laser field is governed by the excited-bound-statepart of the resonance wave function. With an increase in the intensity and by increasing the degree of excitation of the initial state not too high, the electron is ionized at smaller distances from the nucleus.
\end{abstract}

Key words: hydrogen, $2 s$ state, laser field, resonance

\section{INTRODUCTION}

The specific feature of an atom in an external field is that all the bound states decay after some finite lifetime. The ionization may proceed through different mechanisms, which are broadly distinguished according to the value of Keldysh parameter (in atomic units) equal to $\gamma=\left(2 \mathrm{I}_{\mathrm{p}}\right)^{1 / 2} \omega / \hat{\mathrm{E}}_{0}$, where $\mathrm{I}_{\mathrm{p}}$ is the zero field ionization energy of the initial atomic state and $\omega$ and $\hat{E}$ are frequency and field strength of the linearly polarized laser light [1]. For $\gamma>1$, the ionization occurs by the electron which after multiphoton absorption passes through a series of virtual bound levels of the atom (or real levels in the case of the resonant transitions). When the electron is promoted into the continuum, it can continue to absorb photons and to form the higher peaks in the above threshold photoionization spectrum (ATI). The multiphoton ionization is relatively slow process, because the matrix elements of the multiphoton transitions are small [2]. For $\gamma<0.5$ the ionization occurs predominantly as a tunneling or over-the-barrier process [3]. The active atomic electron "sees" the resultant oscillating potential barrier formed by the Coulomb attraction with the nucleus and the instantaneous laser field. Above the "critical" electric

Received December $2^{\text {nd }}, 2012$, accepted January $31^{\text {st }}, 2013$.

${ }^{\dagger}$ Acknowledgement: The author thanks MSc Nenad Milojević for his generous help in preparing this manuscript. The work was supported by the Ministry of Education and Science of the Republic of Serbia under project 171020.

*Coresponding author. E-mail: vucic@ipb.ac.rs. 
field, the electron can pass directly over the potential barrier. During an optical cycle, electron flux will be emitted along the polarization axis first in the one direction, then a half cycle latter in the opposite direction. The emitted electrons are strongly collimated along the polarization direction. The process is rapid, for optical frequencies it can occur during a portion of the optical cycle, once the critical field is reached. The strong field theories postulate that the ground state is coupled only to the continuum by the field and coupling with intermediate states play little or no role. However, Freeman et al. [4] showed when terawatt subpicosecond laser pulses became available, that each ATI peak breaks up into a series of substructures, the so-called Freeman resonances of the ground state with different excited states ponderomotively shifted by the field. There are still unexplained features concerned with the emission of both low-energy and high-energy electrons. For example, the same resonant state may be responsible for peaks of ATI spectrum corresponding to the absorption of small number of photons, as well as to peaks corresponding to large number of absorbed photons. It seems that in strong laser fields some Freeman resonances create conditions favorable for rescattering of the electron by the nucleus, which strongly enhance specific high-kinetic energy regions in the spectrum of the emitted electrons [5].

It is our intention to shed more light into the dynamics of the ionization processes in hydrogen atom initially in $\mathrm{H}(2 \mathrm{~s})$ state exposed to a linearly polarized laser field, using the non-perturbative non-Hermitian Floquet theory [6]. Note that the dynamics of processes involving excited states of the atom differs substantially from that involving the ground state atom, due to closer coupling with other states, including those of the continuum spectrum. The electronic wave function for an atom in a pulsed, high-intensity laser field is in general quite complicated. The electron after it reaches the low continuum oscillates or quivers in response to the field, with a displacement about its equilibrium position equal to $\alpha(\mathrm{t})=-\left(\mathrm{e} / \mathrm{m} \omega^{2}\right) \hat{\mathrm{E}}(\mathrm{t})$, and the quiver amplitude often becomes much larger than the size of the initial orbit. The same is true for the electron in high Rydberg states because of the absence of screening by the nuclear Coulomb potential. For this reason we study the electronic density of the Floquet wave function versus electron coordinate, under various conditions. Although the dynamics of the wave function is not a physical quantity accessible to direct experimental measurement, its theoretical study allows to obtain important information about the internal processes in the atom [7]. Thus, the "bound state" part of electronic density contains relevant information on the structure of the atomic state and of its coupling with other states. The behavior of the wave function in the vicinity of the nucleus where photons can be absorbed is important for understanding the multiphoton ionization and photoemission processes.

\section{THEORY}

Let us consider an atom in the laser field which we treat as a classical monochromatic wave of the constant intensity, spatially homogeneous and linearly polarized, with the vector potential given by $\mathbf{A}=-\mathbf{A}_{0} \sin (\omega \mathrm{t})$. A free electron oscillates in the field and the cycle-averaged kinetic energy of the electron that has zero drift velocity is the ponderomotive energy $\mathrm{U}_{\mathrm{p}}=\mathrm{e}^{2} \hat{\mathrm{E}}^{2} /\left(4 \mathrm{~m} \omega^{2}\right)$.

The dressed states of an atom are described by the solutions of the Schrödinger equation of the form [6] 


$$
\Psi(t)=e^{-i E t / \hbar} \sum_{N} e^{-i N \omega t} F_{N}
$$

The index $\mathrm{N}$ characterizes the total number of real and virtual photons absorbed by the atom. In the velocity gauge, the (time-independent) harmonic components $\mathrm{F}_{\mathrm{N}}$ satisfy the following system of coupled equations

$$
\left(E+N \hbar \omega-H_{\mathrm{at}}\right) F_{N}=V_{+} F_{N-1}+V \cdot F_{N+1}
$$

where $\mathrm{H}_{\mathrm{at}}$ is the Hamiltonian of the field-free atom, and the interaction of the atom with the external field is given by (the atomic units will be used throughout)

$$
V(t)=\mathbf{A}(t) \cdot \mathbf{p}
$$

In Floquet Eq. (2), $V(t)$ is separated into two parts, $V(t)=V_{+} e^{-i \omega t}+V_{-} e^{i \omega t}$, corresponding to the absorption and the emission of a single photon, where $\mathrm{V}_{+}=\mathrm{i} \mathbf{A}_{0} \cdot \mathbf{p} / 2$ and $\mathrm{V}_{-}=\mathrm{V}_{+}^{\dagger}$. Note that we have omitted the term with $\mathrm{A}^{2}$ from $\mathrm{V}(\mathrm{t})$. In the dipole approximation this term does not depend on the position and can be removed by a simple gauge transformation. With this choice of $\mathrm{V}(\mathrm{t})$, the threshold energy of the continuous spectrum of the atom does not shift with an increase of the field intensity, while the ground state is shifted downward by roughly the ponderomotive energy $-U_{p}$. At certain intensity, called the n-photon ionization threshold, the electron acquires just enough of the ponderomotive energy to be promoted into the low continuum by the absorption of $\mathrm{n}$ photons.

The solutions describing dressed bound states decaying by multiphoton ionization are obtained in the position space by imposing the Siegert boundary conditions on the harmonic components [8]. The harmonic components are regular at the nucleus and behave as a superposition of outgoing waves at $r \rightarrow \infty$

$$
F_{N}(\mathbf{r}) \sim \sum_{M} f_{M N} r^{i \gamma_{M}^{c}} e^{i k_{M} r} / r
$$

where $\gamma_{M}^{\mathrm{c}}=\mathrm{Z}_{1} \mathrm{Z}_{2} / k_{M}$ takes into account the logarithmic Coulomb distortion, and M characterizes the number of real photons absorbed by the atom. The wave number of the M-th channel is given by

$$
k_{M}=(2(E+M \omega))^{1 / 2}
$$

The branch of the square root function in equation (5) is chosen so that $\operatorname{Re} \mathrm{k}_{\mathrm{M}} \geq 0$ for $\operatorname{Re}$ $(E+M \omega) \geq 0$, and $\operatorname{Im} k_{M} \leq 0$, or $\operatorname{Im} k_{M} \geq 0$ for $\operatorname{Re}(E+M \omega) \leq 0$. Thus, the Floquet components decrease exponentially at large distances in closed channels (when the electron does not absorb enough photons to be promoted into the continuum) and explode exponentially in the open channels [8].

We solve the Floquet equation (2), by expanding the harmonic components on a discrete basis of spherical harmonics and of discrete basis of complex Sturmian functions, $S_{n l}^{k}$

$$
F_{N}(\mathbf{r})=\sum_{n l} c_{n l}^{N} S_{n l}^{\kappa}(r) \frac{1}{r} Y_{l 0}(\Omega)
$$

Because the problem atom + field has the azimuthal symmetry around the field axis, $\mathrm{L}_{\mathrm{z}}$ is a constant of motion, and we take the magnetic quantum number $\mathrm{m}=0$. 
The Sturmian functions are well adapted to the internal symmetries of the Coulomb potential and to its singularity at the origin. The complex parameter $\mathrm{k}$ of the Sturmian basis is defined by the energy $\mathrm{E}_{\mathrm{st}}=-|\mathrm{K}|^{2} / 2$, close to the initial atomic energy, and by the angle $\theta_{\mathrm{r}}=\pi / 2-\arg (\mathrm{\kappa})$. The choice of the parameter $\mathrm{K}$ in the first quadrant of the complex $\mathrm{k}$ plane implements implicitly the Siegert boundary conditions. Indeed, the functions regular at the origin, which behave like the right hand side of equation (4), for $r \rightarrow \infty$, can be expanded in the basis with decreasing coefficients for increasing order of the expansion, only if the parameter $\mathrm{K}$ lies in the upper-right quadrant of the complex k plane [6]. This choice provides an analytic continuation of the matrix eigenvalue system of equations (2), beyond the real energy axis onto the second sheet of the complex energy plane. The resonance energy is obtained as one of the complex eigenvalues of the analytically continued matrix eigenvalue equation. The complex quasienergy of an atomic level $i$ is given by, $\mathrm{E}_{\mathrm{i}}=\mathrm{E}_{0}+\Delta_{\mathrm{i}}-i \Gamma_{\mathrm{i}} / 2$, where $\mathrm{E}_{0}$ is the field-free energy, $\Delta_{\mathrm{i}}$ is a.c. Stark shift of the level $i$ which tends to zero when the field tends to zero, while $\Gamma_{\mathrm{i}}$ is the induced energy width and (when divided by $\hbar$ ) the total ionization rate from level $i$. The system of equations is solved numerically by inverse iteration method, or by Arnoldi iterations which permits to find a number of closely-spaced energy levels [6]. By expanding the Floquet ansatz over a large basis set with 90 Sturmian functions with angular momentum ranging up to 20 , and with the photon index $\mathrm{N}$ ranging from -20 to 40 , we have been able to obtain convergent quasienergies for excited states of hydrogen.

The temporal dependence of electronic density of quasi-energy initial state versus electron coordinate is given by (7)

$$
|\Psi(r, \theta, t)|^{2}=e^{2 I m E t}\left\{\operatorname{Re}\left[\sum_{N n l} \frac{S_{n l}^{\kappa}(r)}{r} Y_{l 0}(\Omega) c_{n l}^{N} \cos (N \omega t)\right]^{2}+\operatorname{Re}\left[\sum_{N n l} \frac{S_{n l}^{\kappa}(r)}{r} Y_{l 0}(\Omega) c_{n l}^{N} \sin (N \omega t)\right]^{2}\right\}
$$

Note that the velocity $\mathbf{A} \cdot \mathbf{P}$ gauge is appropriate for performing calculations on a basis of Sturmian functions. The electronic density is gauge invariant since it is defined as modulus squared of the wave function and the corresponding ones in $\mathbf{A} \cdot \mathbf{P}$ and $\hat{\mathbf{E}} \cdot \mathbf{R}$ gauges differ only by a field-dependent phase factor.

We have calculated the electronic density along the direction parallel to the field polarization. The variation of this quantity during an optical cycle for a flat part of a long laser pulse of various intensities has been investigated.

\section{ELECTRONIC DENSITY}

If we consider an atom in a laser field whose intensity is not too high to perturb appreciably the internal levels of the atom, the electronic density at small distance from the nucleus must be dominated by the initial state density, i.e. decays more or less exponentially with the radius. At large distance, because the quasi-energy has a negative imaginary part, the probability density for the ionized electron must increase exponentially (see also equation (4)). Thus, in between, the density must have a minimum [8]. The minimum is essentially related to the place of ionization, since for larger radius only the continuum part of the density increases exponentially. As the ionization probability is increasing with field strength, the exponentially increasing part at large distance must also increase with field strength and, consequently, the minimum must shift to smaller distances with the intensity. 
The appearance of the minimum is related only to the exponentially exploding Siegert boundary conditions. However, the effect may be explained on the physical grounds. At low field intensity the electron in the ground state (or in a low-excited state) may be ionized by tunneling through resultant potential barrier formed by the Coulomb attractive potential and the external field. Since the barrier is lowered only slightly by the field, the tunneling ionization is governed by the asymptotic part of the ground- (or the low-excited-) state wave function. The barrier is further lowered with increase of the intensity which causes the place of ionization to approach the nucleus. On the other hand, at higher intensities the electron is likely excited to one (or more) intermediate state(s) before being ionized. The wave function of an excited state shows polynomial character with slow exponential decay, so that the minimum between the bound-excited and the continuum-part of the density is often hard to locate. Furthermore, due to the fact that the Floquet wave function is an eigenstate corresponding to a complex quasi energy, the minimum probably does not represent the "realistic" point of ionization. We expect that our results represent only the correct qualitative behavior of the atomic electron in the process of ionization [9].

The continuum Coulomb wave function is not well represented in the Sturmian basis. Indeed, if one varies the asymptotic behavior of the basis functions by varying $\arg (\mathrm{K})$, the slope of the exponentially increasing part (which corresponds to ionized electron) of the electronic density varies. This is certainly due to the truncation of the basis in the calculation.

There exists a region of stability of the density for laser intensities under study, i. e. for our basis the choice $\theta_{\mathrm{r}}=20^{\circ}$ or $25^{\circ}$, provides the stable bound state part. With this choice, all the above conclusions concerning the general behavior of the density are valid. Moreover, the variation of the slope of continuum part of electronic density with $\arg (\mathrm{K})$, may serve to distinguish it from more stable bound state part. Note that the quasienergies are stable with respect to variation of both $\mathrm{E}_{\mathrm{st}}$ and $\arg (\mathrm{K})$ for a wide "reasonable" region of variation of these parameters. One should note that for a weak non-resonant intensity, the quasi-energy has small but unstable imaginary part with respect to variation of $\arg (\mathrm{K})$ (this affects also the distance of the place of ionization from the nucleus). The results presented are obtained for $\mathrm{E}_{\mathrm{st}}=-0.5$ a.u. and for $\theta_{\mathrm{r}}=25^{\circ}$.

Fig. 1 The Floquet probability density $|\Psi(\mathrm{r}, \theta=0, \mathrm{t}=0)|^{2}$ for the

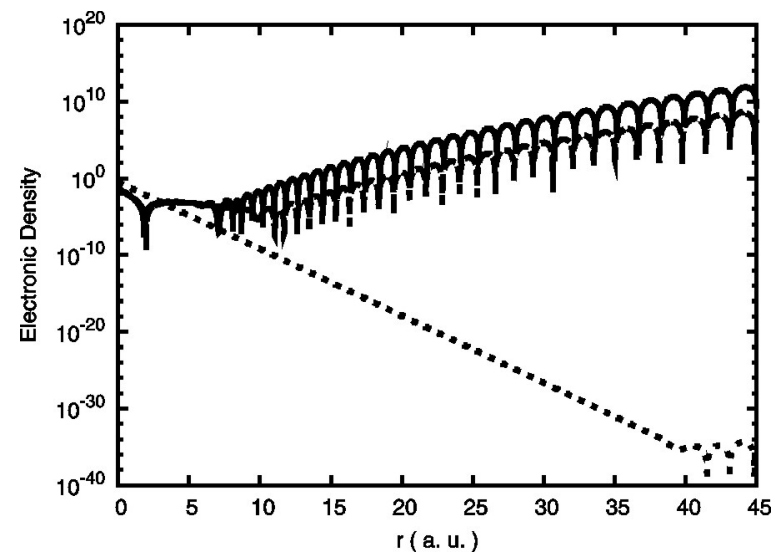
electron ejected from hydrogen in the forward direction (parallel to the laser electric field polarization) at the beginning, $\mathrm{t}=$ 0 , of an optical cycle. The wavelength of the laser field of $800 \mathrm{~nm}$, and (1) the electron is ejected from the $\mathrm{H}(2 \mathrm{~s})$ state at the field intensity of $10^{11} \mathrm{~W} / \mathrm{cm}^{2}$ (solid curve); (2) same as in (1), but for the intensity of $10^{9} \mathrm{~W} / \mathrm{cm}^{2}$ (longdashed curve); (3) the electron is ejected from the $\mathrm{H}(1 \mathrm{~s})$ state at the intensity of $10^{9} \mathrm{~W} / \mathrm{cm}^{2}$ (shortdashed curve). 
Let us study the electronic density in the laser field of $800 \mathrm{~nm}$. The electronic density of the ground state of $\mathrm{H}$ atoms in a low intensity laser field is little affected by the variation of the phase of the field. The density is rather similar to the density of the bound state atom up to a large radius. Thereafter, the electronic density shows regular oscillatory behavior corresponding to the wave function of the ionized electron. For the ground state atom, the electron survives many optical cycles before escaping (the energy width for intensity of $10^{9} \mathrm{~W} / \mathrm{cm}^{2}$ is very small, of the order $10^{-17}$ a.u.). The density corresponding to $H(2 s)$ state shows similar qualitative behavior. For the intensity of $10^{9} \mathrm{~W} / \mathrm{cm}^{2}$, the ionization rate is $10^{-13}$ a.u., and the electron is ionized at much smaller distances from the nucleus. For the intensity $10^{11} \mathrm{~W} / \mathrm{cm}^{2}$, the ionization rate is $10^{-7}$ a.u., and the place of ionization still more approaches the nucleus. We conclude that, at least for the low excited states (with not too high $\mathrm{n}$ and 1 ), the place of ionization approaches the nucleus with increasing field intensity and with the degree of atomic excitation.

Before proceeding further, let us comment about the resonance behavior of atomic states in a laser field. The resonances occur at intensities where the real parts of quasi-energies of two states differ by very nearly an integral multiple, $m$, of the photon energy. If not forbidden by selection rules, these two states are then coupled through the absorption or emission of $\mathrm{m}$ photons; the coupling matrix element is $\hbar \Omega_{0}$, where $\Omega_{\mathrm{o}}$ is the Rabi frequency. For resonances associated with avoided crossing, the real adiabatic (continuous) eigenvalue curves repel one another as the laser intensity, at a fixed wavelength, or conversely, sweeps through the resonance.

If the intensity evolution is slow enough on the time scale $1 / \Omega_{0}$, the atom follows the adiabatic level that originates from the initial level; the atomic characters of the two adiabatic states interchange at a crossing (we mean by "atomic character" the superposition of unperturbed atomic states which approximate the closed-channel part of the Floquet wave function). In the case of diabatic evolution the atom keeps its character. There is no distinction between adiabatic and diabatic evolution for resonances associated to direct crossing, and the atom keeps the initial character as laser intensity sweeps through the resonance. Here, we suppose that the excited states are evolved adiabatically with the laser field wavelength, at a fixed intensity.

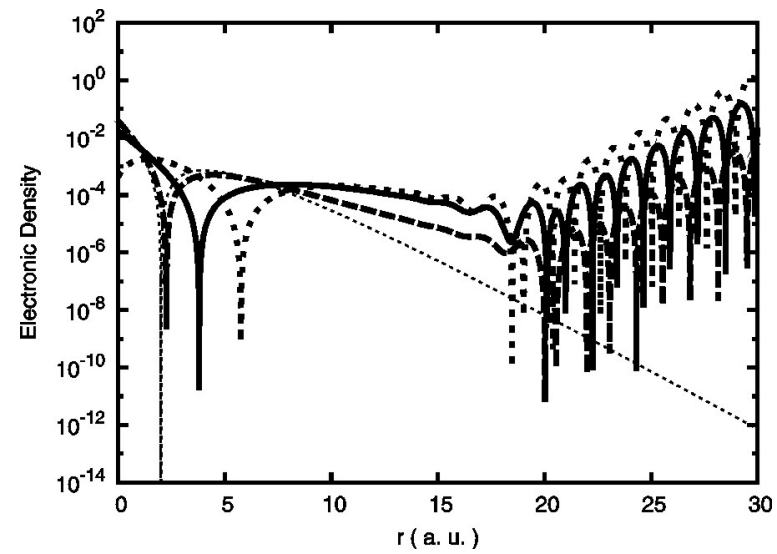

Fig. 2 The electronic density in forward direction at the beginning, $\mathrm{t}$ $=0$, of an optical period, $\mid \Psi(\mathrm{r}, \theta=0, \mathrm{t}$ $=0)\left.\right|^{2}$, for hydrogen in the $2 \mathrm{~s}$ state in the laser field of the intensity of $10^{9}$ $\mathrm{W} / \mathrm{cm}^{2}$, whose wavelength evolves adiabatically throughout the onephoton $2 s-3 p$ resonance, from (1) the non-resonant wavelength of 650 nm (long-dashed curve); to (2) the intermediate resonant wavelength of $656.5 \mathrm{~nm}$ which matches the $2 \mathrm{~s}$ state into one-photon resonance with $3 p$ state (solid curve); and finally to the non-resonant wavelength of $670 \mathrm{~nm}$, with hydrogen evolved adiabatically to dressed state with $3 p$ character (short-dashed curve); dotted curve corresponds to the field-free $2 \mathrm{~s}$ state. 
We can follow clearly from Fig. 2 the evolution of the electronic density from that corresponding to $2 s$ dressed state of hydrogen, to the resonant admixture of $2 s+3 p$ states, and finally to the dressed $3 p$ state.

Note that the minimum of the density corresponding to zero of the wave function with $2 \mathrm{~s}$ character (at $r=2$ a.u.), moves continuously to zero of the $3 p$ wave function, at $r=6$ a.u. Also, the character of the electronic density at relatively small values of radius evolves continuously from the character of the $2 \mathrm{~s}$ state to that of $3 \mathrm{p}$ state. Finally, the electron is ionized at smaller distances from the nucleus with increasing excitation of the atomic states considered.

\section{CONCLUSIONS}

Our conclusion is that the ionization of an atom in a low intensity non-resonant laser field is governed by the asymptotic part of the initial-state wave function. At higher intensities, the initial state is coupled to a higher state(s). This coupling is responsible for the mechanism of interchange of the characters of the two states. The process of the electron emission is governed by the excited-state-part of Floquet wave function corresponding to the final state.

With the increase of intensity and with increasing degree of excitation of the final atomic state, the electron is ionized at smaller distances from the nucleus as a result of lowering of the resultant potential barrier formed by the Coulomb potential and the external field, which "sees" the atomic electron.

\section{REFERENCES}

1. R.M. Potvliege, E. Meşe and S. Vučić, Multiphoton ionization and multiphoton resonances in the tunneling regime, Physical Review A, 81 (5), 053402 (2010).

2. K. Burnett, V.C. Read and P.L. Kneight, Atoms in ultra-intense laser fields, Journal of Physics B: Atomic Molecular and Optical Physics, 26 (4), 561-598 (1993).

3. N.B. Delone and V.P. Krainov, Multiphoton processes in atoms, Springer-Verlag, Berlin, 1994.

4. R.R. Freeman, P.H. Bucksbaum, H. Milchberg, S. Darack, D. Schumacher and M.E. Geusic, Abovethreshold ionization with subpicosecond laser pulses, Physical Review Letters, 59 (10), 1092-1095 (1987).

5. R.M. Potvliege and S. Vučić, High-order above-threshold ionization of argon: Plateau resonances and the Floquet quasienergy spectrum, Physical Review A, 74 (2), 023412 (2006).

6. R.M. Potvliege, STRFLO: A program for time-independent calculations of multiphoton processes in oneelectron atomic systems I. Quasienergy spectra and angular distributions, Computer Physics Communications, 114 (1-3), 42-49 (1998).

7. A. Buchleitner, B. Grémaud and D. Delande, Wavefunctions of atomic resonances, Journal of Physics B: Atomic Molecular and Optical Physics, 27 (13), 2663-2679 (1994).

8. Y.K. Ho, The method of complex coordinate rotation and its applications to atomic collision processes, Physics Reports, 99 (1), 1-68 (1983).

9. S. Vučić, Resonances induced in $\mathrm{H}$ atoms by the high-intensity $800 \mathrm{~nm}$ laser field, Journal of Physics B: Atomic Molecular and Optical Physics, 45 (18), 185601 (2012). 


\section{ATOM VODONIKA U 2S STANJU U LASERSKOM POLJU}

\section{Svetlana Vučić}

Atom vodonika u pobuđenom stanju 2 s nalazi se u laserskom polju. Izučavan je primenom neperturbativne ne-Hermitske teorije Floke. Zaključeno je da sa povećanjem intenzita polja i sa povećanjem stepena pobuđenja početnog stanja, elektron biva jonizovan pri sve manjim rastojanjima od jezgra.

Ključne reči: vodonik, 2 Stanje, lasersko polje, rezonanca 